\title{
Effect of operating conditions on a heavy truck ride comfort with hydro-pneumatic suspension system
}

\author{
Le Xuan Long ${ }^{1}$, Dang Viet $\mathrm{Ha}^{2}$, Le Van Quynh ${ }^{1 *}$, Bui Van Cuong ${ }^{1}$, and Vu Thanh $\mathrm{Niem}^{2}$ \\ ${ }^{1}$ Faculty of Automotive and Power Machinery Engineering, Thai Nguyen University of Technology, Thai Nguyen, Vietnam \\ ${ }^{2}$ Vietnam Register, Ha Noi 12059, Viet Nam
}

\begin{abstract}
The purpose of this paper is to analyze the performance of the hydro-pneumatic suspension system (HPSs) of a mining dump truck on ride comfort under operating conditions. To achieve goals, a 3-D full-vehicle vibration model of a mining dump truck with 10 degrees of freedom is set up to analyze the effects. A nonlinear mathematical model is set up based on the nonlinear characteristics of the HPSs to determine their vertical force which is connected with a 3-D full-vehicle vibration model. The effects of operating conditions on a heavy truck ride comfort are respectively analyzed through the values of the root mean square of acceleration responses of the vertical cab, pitch and roll angles of cab $\left(a_{w c}, a_{w p h i}\right.$ and $\left.a_{w t e t a}\right)$. The analysis results indicate that the survey conditions have a great influence on vehicle ride comfort. Especially, the values of $\mathrm{a}_{\mathrm{wc}}, \mathrm{a}_{\mathrm{wphi}}$ and $\mathrm{a}_{\text {wteta }}$ with the poor road surface condition respectively reduce by $43.1 \%, 45.9 \%$ and $61.8 \%$ compared to the very poor road surface condition at vehicle speed of $30 \mathrm{~km} / \mathrm{h}$ and full load.
\end{abstract}

\section{Introduction}

With its outstanding advantages, hydro-pneumatic suspension system (HPSs) is increasingly used on vehicles, especially heavy trucks that often operate on off-road surfaces. Due to the viscous resistance of the fluid and the compressibility of gas the damping and stiffness coefficient of the HPSs are adjusted when vehicles operate with different conditions. Co-simulation technique was used to study the factors affecting the safety and ride comfort of vehicle movement. Through simulating the influence of vehicle, cylinder diameter and pressure of tank of HPSs on movement safety was investigated and quantified [1]. To improve the ride comfort performance of vehicle, the particle swarm algorithm (PSO) was applied to find the optimal parameters of HPSs of an off-road vehicle and the fuzzy controller was used to control the optimal design parameters [2-3]. The HPSs model parameters are optimized with the systematic objective function to improve ride performance of the HPS [4]. The air part of the HPSs combines an air tank and an auxiliary air chamber with its height is controlled using a fuzzy controller to improve the ride comfort of vehicle [5]. The performance of the HPSs of heavy truck on the ride quality of road surfaces analyzed and compared with other types of suspension systems using 3-D full-vehicle vibration model [6-7]. The traditional HPSs with extra an accumulator through by a controllable throttle valve was studied and the appropriate selection of the HPSs model parameters were optimized [8]. The effect of operating conditions on road surface were analyzed and evaluated through the values of the dynamic load factor of the wheel using 3-D full-vehicle vibration model [9]. The effectiveness of two types of heavy truck suspension systems in terms of reducing the dynamic wheel forces acting on road surface was proposed and compared under different operating conditions 3-D full-vehicle vibration model [10]. The objective of this work is to analyze the performance of the HPSs of a mining dump truck on ride comfort under operating conditions. A 3-D full-vehicle vibration model of a mining dump truck with 10 degrees of freedom is set up to analyze the effects. A nonlinear mathematical model is set up based on the nonlinear characteristics of the HPSs to determine their vertical forces which is connected with a 3-D full-vehicle vibration model. The vibration equations are solved by Matlab/Simulink software. The effects of operating conditions on a heavy truck ride comfort are respectively analyzed through the values of $\mathrm{a}_{\mathrm{wc}}, \mathrm{a}_{\mathrm{wphi}}$ and $\mathrm{a}_{\mathrm{wteta}}$ are chosen as objective functions.

\section{Three Dimensional Full-Vehicle Vibration Model}

A structural diagram of a mining dump truck is chosen to analyze the performance of HPSs on ride comfort under operating conditions, as shown in Figure 1. A 3-D full-vehicle vibration model of a mining dump truck with 10 degrees

${ }^{*}$ Corresponding author: lequynh@tnut.edu.vn 
of freedom (DOF) using HPSs is shown in Fig.2, where, $\mathrm{z}_{\mathrm{c}}, \varphi_{\mathrm{c}}$ and $\theta_{\mathrm{c}}$ are the vertical and angular displacements of the $\mathrm{cab}$, respectively are $z_{c}, \varphi_{c}$ and $\theta_{c}$, respectively, $z_{b}, \varphi_{b}$ and $\theta_{b}$ are the vertical and angular displacements of the vehicle body, respectively, $\mathrm{z}_{\mathrm{a} 1}, \theta_{\mathrm{a} 1}$ and $\mathrm{z}_{\mathrm{a} 2}, \theta_{\mathrm{a} 2}$ are the vertical and angular displacements of the $1^{\text {st }}$ axle and $2^{\text {nd }}$ axle, respectively, $\mathrm{m}_{\mathrm{a} 1}, \mathrm{~m}_{\mathrm{a} 2}, \mathrm{~m}_{\mathrm{b}}, \mathrm{m}_{\mathrm{c}}$ are $1^{\mathrm{st}}$ axle, $2^{\mathrm{nd}}$ axle vehicle and cab masses, respectively, $\mathrm{I}_{\mathrm{ax} 1}, \mathrm{I}_{\mathrm{ax} 2}, \mathrm{I}_{\mathrm{bx}}, \mathrm{I}_{\mathrm{by}}, \mathrm{I}_{\mathrm{cx}}, \mathrm{I}_{\mathrm{cy}}$ are the inertia moments of $1^{\text {st }}$ axle, $2^{\text {nd }}$ axle vehicle and cab masses, respectively, $\mathrm{k}_{\mathrm{ti}}$ and $\mathrm{c}_{\mathrm{ti}}$ are stiffness and damping coefficients of tires, respectively, $\mathrm{k}_{\mathrm{i}}$ and $\mathrm{c}_{\mathrm{i}}$ are stiffness and damping coefficients of HPSs, respectively, $\mathrm{k}_{\mathrm{ci}}$ and $\mathrm{c}_{\mathrm{ci}}$ are stiffness and damping coefficients of cab mount system, respectively, $1_{k}$ and $b_{i}$ are the distances and $q_{i}$ are the excitation functions of road surface $(\mathrm{i}=1 \div 4, \mathrm{k}=1 \div 7)$.
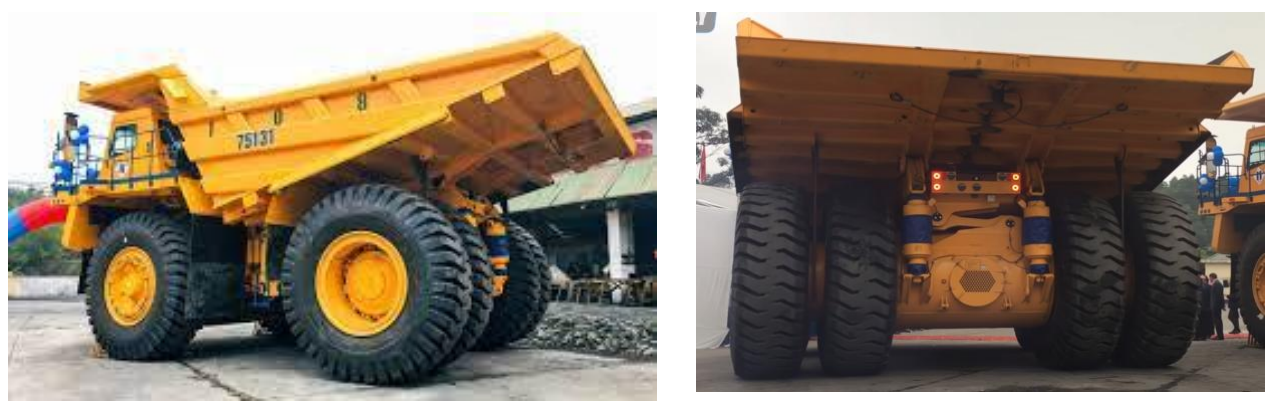

Fig. 1. Structural diagram of a mining dump truck
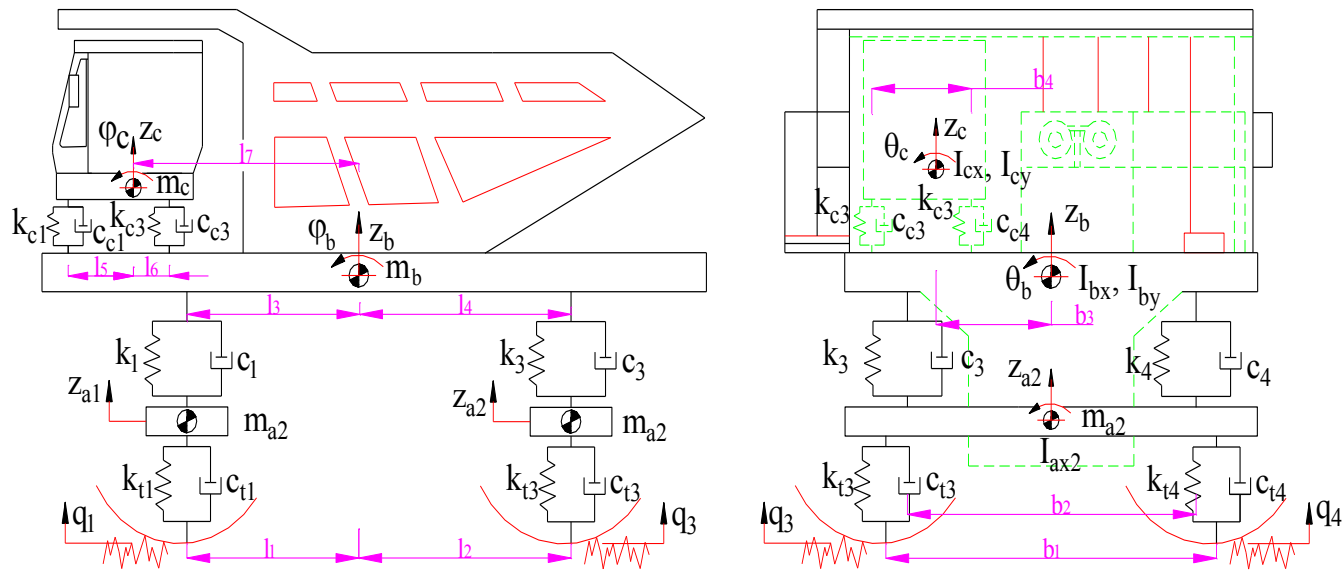

Fig. 2. A 3-D full-vehicle vibration model of a mining dump truck

The theory of multi-body and D'Alembert's principle are used to establish the motion equations of the dynamic systems of a mining dump truck in Figure 2. The motion equations for the dynamic systems of a mining dump truck is given by the matrix form

$$
[M]\{\ddot{u}\}=[F]
$$

where, $[\mathrm{M}]$ is the matrix of mass: $[\mathrm{M}]=\left[\mathrm{A}_{\mathrm{ij}}\right],(\mathrm{i}, \mathrm{j}=1 \div 10)$. If $\mathrm{i} \neq \mathrm{j}$ then $\mathrm{A}_{\mathrm{ij}}=0$ and if $\mathrm{i}=\mathrm{j}$ then $\mathrm{A}_{\mathrm{ij}}$ is defined as

$$
\left(A_{i j}\right)=\left(m_{c}, m_{b}, m_{a 1}, m_{a 2}, I_{a x 1}, I_{a x 2}, I_{b x}, I_{b y}, I_{c x}, I_{c y}\right)
$$

$\{\mathrm{u}\}$ is the vector of displacement which is defined as

$$
\{u\}=\left\{z_{c}, z_{b}, z_{a 1}, z_{a 2}, \varphi_{c}, \varphi_{b}, \theta_{c}, \theta_{b}, \theta_{a 1}, \theta_{a 2}\right\}^{T}
$$

$[\mathrm{F}]$ is the matrix of the vertical force and moment in motion direction of $z, \varphi$ and $\theta$ at the center of gravity of $1^{\text {st }}$ axle, $2^{\text {nd }}$ axle, vehicle body and cab which is defined as.

$$
[F]=\left[F_{z}, R\right]^{T}
$$

$\left[\mathrm{F}_{\mathrm{z}}\right]$ is the matrix of the vertical force which is defined as

$$
\left[F_{z}\right]=\left[F_{c i}, F_{i}, F_{t i}\right]^{T}
$$

where, $\mathrm{F}_{\mathrm{ci}}, \mathrm{F}_{\mathrm{i}}, \mathrm{F}_{\mathrm{ti}}$ are the vertical forces acting on cab body, vehicle body, $1^{\text {st }}$ axle and $2^{\text {nd }}$ axle. 
$[R]$ is the matrix of the moment in motion which is defined as

$$
[R]=\left[R_{c \varphi}, R_{b \varphi}, R_{c \theta}, R_{b \theta}, R_{a 1 \theta}, R_{a 2 \theta}\right]^{T}
$$

where, $\mathrm{R}_{\mathrm{c} \varphi}, \mathrm{R}_{\mathrm{b} \varphi}$ are the moment in motion direction of angle $\varphi$ of the cab and vehicle bodies; $\mathrm{R}_{\mathrm{c} \theta}, \mathrm{R}_{\mathrm{b} \theta}, \mathrm{R}_{\mathrm{a} 1 \theta}, \mathrm{R}_{\mathrm{a} 2 \theta}$ are moment in motion direction of angle $\theta$ of the cab, vehicle bodied, $1^{\text {st }}$ axle and $2^{\text {nd }}$ axle.

The excitation functions of road surface: The excitation functions from road surface roughness is represented by various mathematical functions. The random road surface roughness random road profile according to ISO 8608 [11] is selected as excitation functions for vehicle which is defined as

$$
q(t)=\sum_{i=1}^{N} \sqrt{2 G_{d}\left(\Omega_{i}\right) \frac{\Delta \Omega}{\pi}} \cos \left(\Omega_{i} t+\alpha_{i}\right)
$$

where, $G_{d}\left(\Omega_{i}\right)$ is the power spectral density at angular frequency $\Omega, \Delta \Omega_{\mathrm{i}}$ is the variance of the pavement profile which depends on the spatial angular frequency and the time step, $\alpha_{i}$ is the phase of the harmonic function (rad) which is randomly generated between 0 and $\pi$.

\section{Mathematical Model of HPS}

A structural schematic of HPS model is shown in Fig. 3 which consists of the main oil chamber (1) and ring oil chamber (2) and the gas chamber (3), the vertical displacements of vehicle axle, floating piston, and vehicle body, $z_{a}, z_{d}$ and $z_{b}$. A dynamic model of HPSs is developed on the structural diagram of Fig. 3, as shown in Fig. 3 which consists of the stiffness and damping coefficients of HPS $\mathrm{k}$ and $\mathrm{c}$, vehicle axle and vehicle body masses, $\mathrm{m}_{\mathrm{a}}$ and $\mathrm{m}_{\mathrm{b}}$.

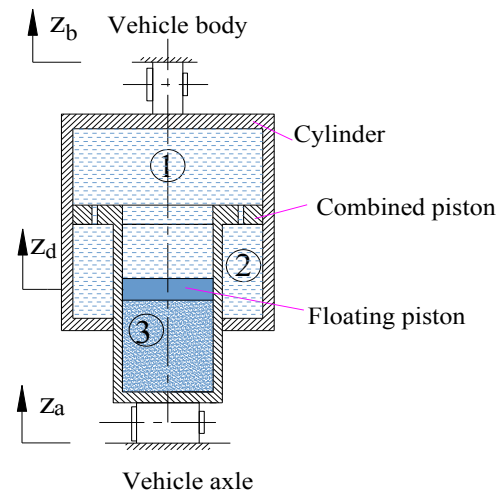

Fig. 3. A structural schematic of HPS

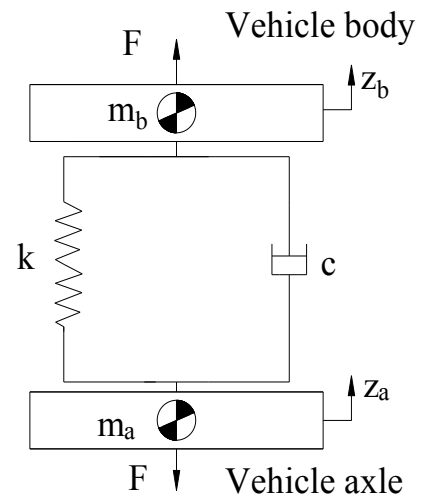

Fig. 4. A dynamic model of HPS

From Fig. 3, the vertical dynamic force of HPSs is determined as:

$$
F=F_{a}+F_{h}
$$

where, $F_{a}, F_{h}$ are the elastic and damping force of HPS.

For the HPSs model (Figure 4), the elastic force of HPS is formed by elasticity of gas in air chamber which is defined by

$$
F_{a}=p_{0}\left[\left(\frac{V_{0}}{V_{0}+A_{p}\left(z_{b}-z_{a}\right)}\right)^{n}-1\right] A_{p}
$$

where, $\mathrm{p}_{0}$ and $\mathrm{V}_{0}$ are the initial absolute pressure and volume in the air chamber (3), $\mathrm{p}_{\mathrm{a}}$ and $\mathrm{V}_{\mathrm{a}}$ are the absolute pressure and volume in the air chamber (3), $A_{p}$ is the area of floating piston, $n$ is the polytrophic rate $(1<\mathrm{n}<1.4)$.

The damping force of the suspension system is formed by the viscous of the oil through the orifices of combined piston which is defined as

$$
F_{h}=p_{1}\left(A_{s}-A_{p}\right)-p_{2}\left(A_{s}-A_{c}\right)-\left(p_{0}-\frac{m_{d} g}{A_{p}}\right)\left(A_{c}-A_{p}\right)
$$

where, $\mathrm{A}_{\mathrm{s}}$ and $\mathrm{A}_{\mathrm{c}}$ are the area of cylinder and rod piston, $\mathrm{p}_{1}$ and $\mathrm{p}_{2}$ are the pressures in chamber (1) and chamber (2). The flow rate through the orifices is defined as

$$
Q=C_{d} A \sqrt{2 \frac{\left(p_{2}-p_{1}\right)}{\rho} \operatorname{sign}\left(\dot{z}_{b}-\dot{z}_{a}\right)} \text {, Other way } Q=\left(A_{s}-A_{c}\right)\left(\dot{z}_{b}-\dot{z}_{a}\right)
$$

where, $C_{d}$ is coefficient of discharge, $A$ is the area of the orifices and $\rho$ is the density of oil.

The flow rate is inferred base on the basic of volume balance. From Eq. (11), the relationship $\mathrm{p}_{1}$ and $\mathrm{p}_{2}$ is achieved as 


$$
p_{2}=p_{1}+\frac{\rho\left(A_{s}-A_{c}\right)^{2}\left(\dot{\mathrm{z}}_{b}-\dot{\mathrm{z}}_{a}\right)^{2} \operatorname{sign}\left(\dot{\mathrm{z}}_{b}-\dot{\mathrm{z}}_{a}\right)}{2 C_{d}^{2} A^{2}}
$$

The differential motion equation of floating piston could be determined as

$$
\left(p_{1}-p_{a}\right) A_{p}-m_{d} g=m_{d} \ddot{z}_{d} \Rightarrow p_{1}=p_{a}-\frac{m_{d}\left(\ddot{z}_{d}+g\right)}{A_{3}}
$$

Combination of Eq. (12), Eq. (13) and Eq. (10), the damping force of HPS is achieved as

$$
\begin{aligned}
& F_{c}=-\frac{\rho\left(A_{s}-A_{c}\right)^{3}\left(\dot{\mathrm{z}}_{b}-\dot{\mathrm{z}}_{a}\right)^{2} \operatorname{sign}\left(\dot{\mathrm{z}}_{b}-\dot{\mathrm{z}}_{a}\right)}{2 C_{d}^{2} A^{2}} \\
& +p_{0}\left(A_{c}-A_{p}\right)\left(\left(\frac{V_{0}}{V_{0}+A_{p}\left(z_{b}-z_{a}\right)}\right)^{n}-1\right)-\frac{m_{d} \ddot{z}_{d}}{A_{p}}\left(A_{c}-A_{p}\right)
\end{aligned}
$$

Combination of Eq. (14) and Eq. (9), the vertical force of HPS is achieved as

$$
\begin{aligned}
& F_{h}=-\frac{\rho\left(A_{s}-A_{c}\right)^{3}\left(\dot{\mathrm{z}}_{b}-\dot{\mathrm{z}}_{a}\right)^{2} \operatorname{sign}\left(\dot{\mathrm{z}}_{b}-\dot{\mathrm{z}}_{a}\right)}{2 C_{d}^{2} A^{2}} \\
& +p_{0} A_{c}\left(\left(\frac{V_{0}}{V_{0}+A_{p}\left(z_{b}-z_{a}\right)}\right)^{n}-1\right)-\frac{m_{d} \ddot{z}_{d}}{A_{p}}\left(A_{c}-A_{p}\right)
\end{aligned}
$$

\section{Results and Discussion}

The motion equations for the dynamic systems of mining dump truck degrees are simulated and calculated the objective functions by using Matlab/Simulink software with vehicle and HPS parameters of the reference [12]. The simulation results of the vertical cab acceleration, pitch and roll angular acceleration of cab when vehicle moves on two survey road surface conditions (ISO class D (poor condition)-Type 1 and ISO class E (very poor condition)-Type 2) at v=30 $\mathrm{km} / \mathrm{h}$ and full load are shown in Figure 5. The obtained results of Figure 5 indicate that the peak amplitude values of of $\mathrm{a}_{\mathrm{c}}, \mathrm{a}_{\mathrm{phi}}$ and $\mathrm{a}_{\text {teta }}$ with on the very poor road surface condition increase compared to the poor road surface condition which lead to improve significantly vehicle ride comfort with good road conditions. The obtained results of Figure 5 could be determined the values of the root mean square of acceleration responses of the vertical cab, pitch and roll angles of cab

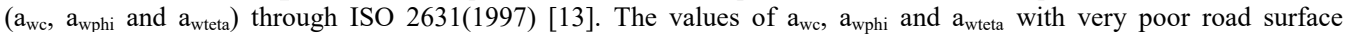
condition (ISO class D) respectively reduce by $43.1 \%, 45.9 \%$ and $61.8 \%$ compared to the very poor road surface condition (ISO class E). The ride performance of the HPSs under operating conditions will be analyzed in the next sections.

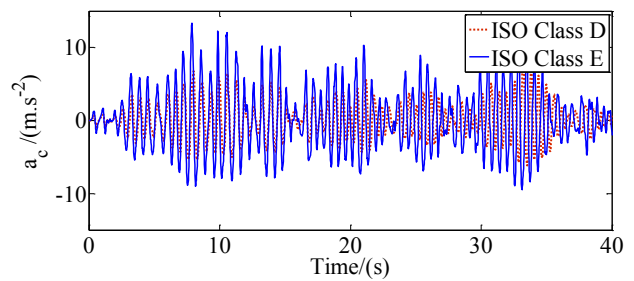

(a) The vertical acceleration at the center of gravity of cab $\left(a_{c}\right)$

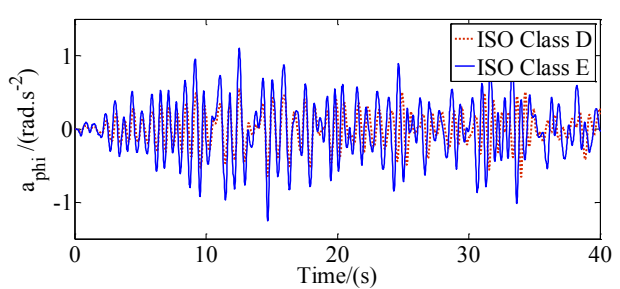

(b) The pitch angle acceleration at the center of gravity of cab $\left(\mathrm{a}_{\mathrm{phi}}\right)$

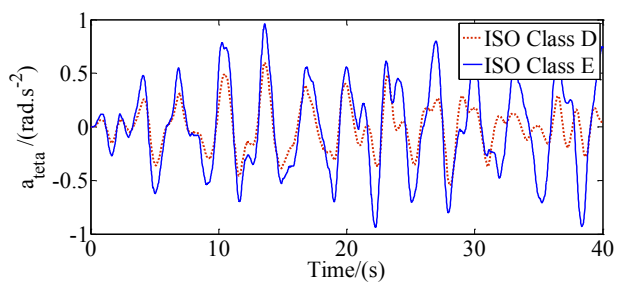

(c) The roll angle acceleration at the center of gravity of cab $\left(a_{\text {teta }}\right)$

Fig. 5. The comparative values of $a_{c}, a_{\text {phi }}$ and $a_{\text {teta }}$ with two survey road surface conditions 


\subsection{Vehicle load conditions}

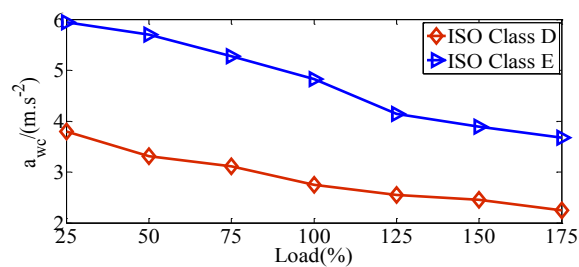

(a) $\mathrm{a}_{\mathrm{wc}}$ values with changing vehicle load

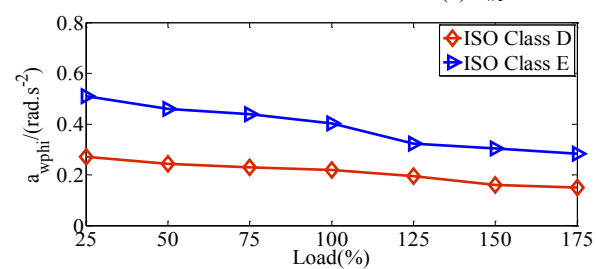

(b) $a_{\text {wphi }}$ values with changing vehicle load

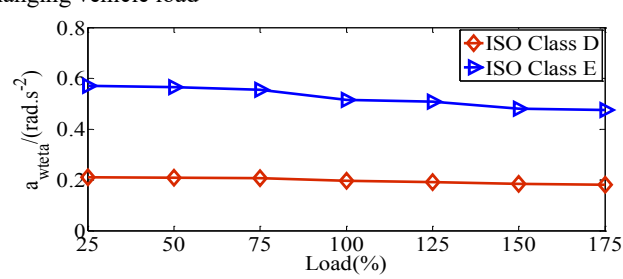

(c) $\mathrm{a}_{\text {wteta }}$ values with changing vehicle load

Fig. 6. The $\mathrm{a}_{\mathrm{cb}}$, $\mathrm{a}_{\mathrm{wphi}}$ and $\mathrm{a}_{\mathrm{wteta}}$ values under changing vehicle load conditions

The $\mathrm{a}_{\mathrm{cb}}, \mathrm{a}_{\mathrm{wphi}}$ and $\mathrm{a}_{\mathrm{wteta}}$ values under changing vehicle load conditions are presented in Fig. 6.

\subsection{Road surface conditions}

In order to analyze the effects of road conditions on vehicle ride comfort with HPSs, five road surface conditions from (ISO class A) to (ISO class E) are chosen when vehicle moves at two survey vehicle speed conditions such as $\mathrm{v}=15$ $\mathrm{km} / \mathrm{h}$ and $\mathrm{v}=30 \mathrm{~km} / \mathrm{h}$ and full load. The $\mathrm{a}_{\mathrm{wc}}, \mathrm{a}_{\mathrm{wphi}}$ and $\mathrm{a}_{\mathrm{wteta}}$ values during the road surface quality changes from very good condition to very poor condition are shown in Fig. 7. The results of Fig. 7 indicate that the $a_{w c}, a_{w p h i}$ and $a_{w t e t a}$ values quickly increase while road surface conditions change badly. On the other hand, the $a_{w c}, a_{\text {wphi }}$ and $a_{\text {wteta }}$ values with at $\mathrm{v}=15 \mathrm{~km} / \mathrm{h}$ compared to $\mathrm{v}=30 \mathrm{~km} / \mathrm{h}$ which lead to the vehicle ride comfort improved under low vehicle speed conditions and good road surface conditions. The quality of the road surface not only greatly affects the ride comfort of the vehicle, but also it greatly affects the part of vehicle and the safe movement of vehicle. However, from the results of the analysis of the effect of the operating conditions on vehicle ride comfort, it shows that the performance of HPSs has been significantly improved vehicle ride comfort under large amplitude excitation region of road surface roughness.

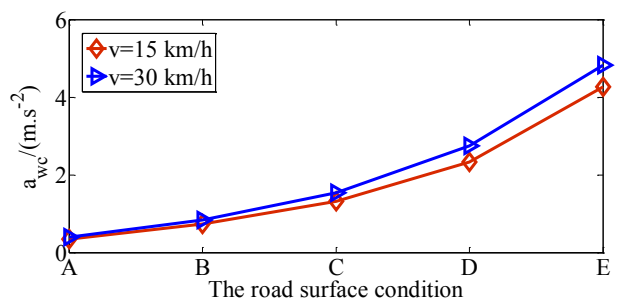

(a) $a_{w c}$ values with changing road surface condition

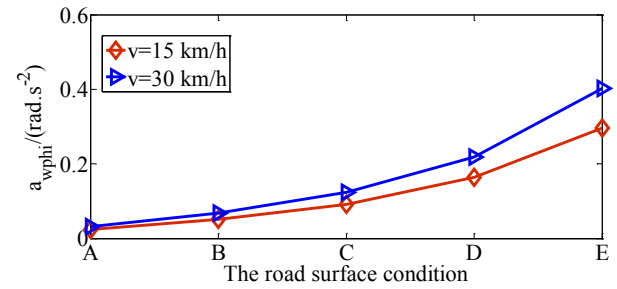

(b) $a_{\text {wphi }}$ values with changing road surface condition

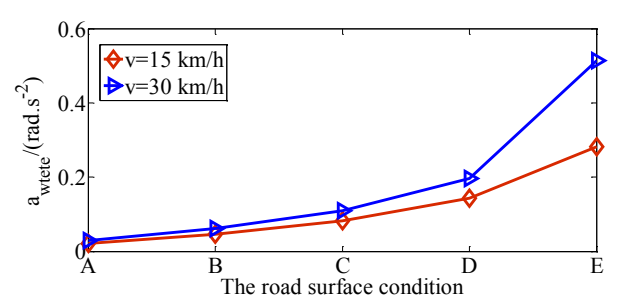

(c) $\mathrm{a}_{\text {wteta }}$ values with changing road surface condition

Fig. 7. The $a_{c b}, a_{w p h i}$ and $a_{\text {wteta }}$ values under changing road surface condition 


\section{Conclusions}

In this study, a 3-D full-vehicle vibration model of a mining dump truck with 10 degrees of freedom was set up to analyze the effects. A nonlinear mathematical model was set up based on the nonlinear characteristics of the HPSs to determine their vertical forces which is connected with a 3-D full-vehicle vibration model. The ride performance of HPSs in the direction of the vehicle ride comfort is analyzed under the operating conditions. Some conclusions can be drawn from the results of the effect analysis: (1) The values of $\mathrm{a}_{\mathrm{wc}}, \mathrm{a}_{\mathrm{wphi}}$ and $\mathrm{a}_{\mathrm{wteta}}$ with very poor road surface condition (ISO class D) respectively reduce by $43.1 \%, 45.9 \%$ and $61.8 \%$ compared to the very poor road surface condition (ISO class E), (2) The values of $\mathrm{a}_{\mathrm{wc}}$ and $\mathrm{a}_{\mathrm{wphi}}$ quickly decrease in the process of increasing the load of the vehicle, meanwhile the values of $a_{w t e t a}$ has changed very little, and (3) the $a_{w c}, a_{w p h i}$ and $a_{w t e t a}$ values quickly increase while road surface conditions change badly. Furthermore, the study results indicate that the performance of HPSs has been significantly improved vehicle ride comfort under large amplitude excitation region of road surface roughness.

\section{Acknowledgment}

The work described in this paper was supported by Thai Nguyen University (TNU), Thai Nguyen University of Technology (TNUT) for a scientific project (Code: DH2019-TN02-02). Occasionally

\section{References}

1. Sh. Han et al., Shock and Vibration (2017)

2. H. Zhao et al., Advances in Mechanical Engineering 10, 11 (2018)

3. B. Gong et al., Advances in Mechanical Engineering 7, 6 (2015)

4. B. Qin et al. Applied Sciences (2021)

5. H.Y. Qi, Proceedings of the Institution of Mechanical Engineers Part D: Journal of Automobile Engineering 2019, (2019)

6. L.X. Long et al., Inter. J. of Mech. Eng. and Tech. 9 (2019)

7. L.X. Long et al., IOP Conf. Ser.: Mater. Sci. Eng. (2020)

8. Zh. Sang et al., Advances in Mechanical Engineering 9, 5 (2017)

9. L.V. Quynh et al., Vibroengineering PROCEDIA 16 (2017)

10. V.Q. Le, Vibroengineering Procedia 14 (2017)

11. ISO 8608, Mechanical Vibration-Road Surface Profiles-Reporting of Measured Data. International Organization for Standardization (1995)

12. L.X. Long, Science research topic, Thai Nguyen University, Thai Nguyen, Vietnam (2021)

13. ISO 2631-1, Mechanical Vibration and Shock-Evaluation of Human Exposure to Whole-Body Vibration, Part I: General Requirements. ISO (1997)

14. V.Q. Le, J. Zhang et al., J. South. Univer. 27 (2011)

15. L.V. Quynh, B.V. Cuong et al., Adv. In Engi. Re and App. (2019) 